\title{
The dimension of metrizable subspaces of Eberlein compacta and Eberlein compactifications of metrizable spaces
}

\author{
by \\ Michael G. Charalambous (Karlovassi)
}

\begin{abstract}
We prove that every Baire subspace $Y$ of $c_{0}(\Gamma)$ has a dense $G_{\delta}$ metrizable subspace $X$ with $\operatorname{dim} X \leq \operatorname{dim} Y$. We also prove that the Kimura-Morishita Eberlein compactifications of metrizable spaces preserve large inductive dimension. The proofs rely on new and old results concerning the dimension of uniform spaces.
\end{abstract}

1. Introduction and brief summary of results. Amir and Lindenstrauss [1] defined Eberlein compact spaces to be those compact spaces that are homeomorphic to weakly compact subsets of Banach spaces. They proved that a space is Eberlein compact iff it is homeomorphic to a compact subspace of $c_{0}(S)$ for some set $S$, where $c_{0}(S)$ is the subspace of the product of unit intervals $\prod_{s \in S} \mathbb{I}_{s}$ consisting of the points $x=\left(x_{s}\right)$ such that $\left\{s \in S: x_{s}>\varepsilon\right\}$ is finite for each positive $\varepsilon$. It is known that every Eberlein compact space contains a dense $G_{\delta}$ subspace which is metrizable. In fact, Dimov [6] has extended this result by showing that every Baire subspace $Y$ of $c_{0}(S)$ contains a dense $G_{\delta}$-subspace $X$ which is metrizable. In Section 4 of this paper, we prove that $\operatorname{dim} X \leq \operatorname{dim} Y$. This is obtained as an application of Theorem 1, the main result of Section 3, which concerns the covering dimension of uniform spaces. Section 4 also contains characterizations of metrizable and Eberlein compact spaces in terms of families of functions generating their topology.

It should be noted that for a dense metrizable subspace $X$ of a compact space $Y$, we do not necessarily have $\operatorname{dim} X \leq \operatorname{dim} Y$. For if $X$ is Roy's example in [13], then $X$ is metrizable and $\operatorname{dim} X=1$ while ind $X=0$. Therefore $X$ has a compactification $Y$ with $\operatorname{dim} Y=0$.

It is also of interest to note that Kimura and Morishita [9] have recently proved that every metrizable space $M$ has an Eberlein compactification $E$

2000 Mathematics Subject Classification: Primary 54F45; Secondary 54E15, 54E35, $54 \mathrm{E} 52$.

Key words and phrases: Eberlein compact and metrizable spaces, covering and inductive dimension of topological and uniform spaces. 
with $\operatorname{dim} E=\operatorname{dim} M$. In Section 5, we show that, in fact, Ind $E=\operatorname{dim} M$. The proof relies heavily on properties of inductive dimensions of uniform spaces.

A very general subset theorem due to Pasynkov [11] asserts that $\operatorname{dim} X \leq$ $\operatorname{dim} Y$ whenever $X$ is a $d$-regular subspace of $Y$. In Section 6 , where all relevant definitions can be found, we show that a metrizable space is a $d$-regular subspace of its Kimura-Morishita compactification. However, in the case of the first result quoted in the abstract, it is not clear whether the metrizable space $X$ is a $d$-regular subspace of $Y$. Our final result is a subset theorem that covers both the case just mentioned as well as the case of $d$-regularity.

For the standard results in General Topology and Dimension Theory, we refer the reader to [7] and [8], respectively.

2. Definitions and prerequisites. In this section, we give the necessary definitions concerning the covering dimension of uniform spaces introduced by the author in [2], and list the results needed in subsequent sections.

The cozero set of a continuous function $f: X \rightarrow Y$ into a metric space with a distinguished element 0 is defined by $\operatorname{coz} f=\{x \in X: f(x) \neq 0\}$. The uniformly open sets of a uniform space are the cozero sets of uniformly continuous functions into the unit interval $\mathbb{I}$. Their complements are called uniformly closed. The dimension Dim of a uniform space $X$ is defined to be the least element $n$ of $\{-1,0,1,2, \ldots, \infty\}$ such that each finite cover of $X$ by uniformly open sets has a finite refinement of order $\leq n$ consisting of uniformly open sets. Evidently, $\operatorname{Dim} X=\operatorname{dim} X$ if every cozero set of $X$ is uniformly open, e.g. if $X$ is a metric space. If the uniformity on $X$ is $\mathcal{U}$, the uniformly open (resp. closed) sets of $X$ may be called $\mathcal{U}$-open (resp. $\mathcal{U}$-closed), and we may write $\mathcal{U}$ - $\operatorname{dim} X$ instead of $\operatorname{Dim} X$, if it is necessary to indicate the uniformity. Also, by abuse of notation, we use $\mathcal{U}$ instead of $\mathcal{U}_{Y}$ for the subspace uniformity on any subset $Y$ of $X$.

It should be remarked that the collection of uniformly open sets is closed with respect to taking finite intersections and countable unions, and inverse images under uniformly continuous functions of uniformly open sets (such as open sets in a metric space) are uniformly open. Also, the uniformly open sets of a subspace $Y$ of $X$ are exactly the traces on $Y$ of the uniformly open sets of $X$.

Dim has a number of interesting features, and we proceed to list those needed in what follows. The first two results are taken from [2].

Proposition 1 (The subset theorem). For any subspace $Y$ of a uniform space $X, \operatorname{Dim} Y \leq \operatorname{Dim} X$. 
Proposition 2 (The countable sum theorem). Let $X$ be the countable union of uniformly closed subspaces with $\operatorname{Dim} \leq n$. Then $\operatorname{Dim} X \leq n$.

COROLlary 1. Let $X$ be the countable union of subspaces each of which is either uniformly closed or uniformly open and has $\operatorname{Dim} \leq n$. Then $\operatorname{Dim} X$ $\leq n$.

Proof. This follows from Propositions 1 and 2 on noting that each uniformly open set of a space $X$ is the union of countably many uniformly closed subspaces of $X$.

COROllary 2. Let $X$ be the union of uniformly closed subspaces $F_{1}, F_{2}, \ldots$ such that $\operatorname{Dim}\left(F_{i} \backslash F_{i-1}\right) \leq n$ for each $i \in \mathbb{N}$, where $F_{0}=\emptyset$. Then $\operatorname{Dim} X \leq n$.

Proof. We first apply induction to get $\operatorname{Dim} F_{i} \leq n$. Indeed, if $\operatorname{Dim} F_{i-1}$ $\leq n$, then by Corollary $1, \operatorname{Dim}\left(\left(F_{i} \backslash F_{i-1}\right) \cup F_{i-1}\right) \leq n$. Hence, by Proposition $1, \operatorname{Dim} F_{i} \leq n$. Now, by Proposition $2, \operatorname{Dim} X \leq n$.

The following is essentially Theorem 4 of [5]. We have added the last sentence whose truth is transparent from the proof.

Proposition 3. Let $A$ be a subspace of a uniform space $X$ with $\operatorname{Dim} A$ $\leq n$, and $f: X \rightarrow Y$ a uniformly continuous function into a metric space. Then there are uniformly continuous functions $g_{i}: X \rightarrow \mathbb{I}, i \in \mathbb{N}$, such that if $h=f \Delta \triangle\left\{g_{i}: i \in \mathbb{N}\right\}: X \rightarrow Y \times \mathbb{I}^{\mathbb{N}}$, then $\operatorname{dim} h(A) \leq n$. If $G_{i}$ is a uniformly open set of $X$ containing $A, i \in \mathbb{N}$, then $g_{i}$ may be chosen so that $\operatorname{coz} g_{i} \subset G_{i}$.

Finally, we will need the following result from [4].

Proposition 4. Let $(X, \mathcal{U})$ be the limit of an inverse system $\left(X_{\alpha}, \mathcal{U}_{\alpha}\right.$, $\left.p_{\alpha, \beta}\right)$ of uniform spaces with $\mathcal{U}_{\alpha}-\operatorname{dim} X_{\alpha} \leq n$. Then $\mathcal{U}-\operatorname{dim} X \leq n$.

3. Locally finite collections and dimension. Let $S$ be a set and $X$ a metric space with metric $\varrho$ and a distinguished point 0 . Let $X(S)$ be the the union of all $X \times\{s\}, s \in S$, where all points $(0, s)$ have been identified to a single point 0 . For each $s \in S$, let $X_{s}$ denote the image of $X \times\{s\}$ under this identification and let $\varrho_{s}$ be the metric on $X_{s}$ induced by $\varrho$ in the obvious way. The hedgehog with $|S|$ spines homeomorphic to $X$ is $X(S)$ with metric $d$ defined by $d(x, y)=\varrho_{s}(x, y)$ if $x, y \in X_{s}$, and $d(x, y)=\varrho_{s_{1}}(x, 0)+\varrho_{s_{2}}(y, 0)$ if $x \in X_{s_{1}}, y \in X_{s_{2}}$ and $s_{1} \neq s_{2}$.

With the notation just established, for a cardinal $\tau, J(\tau)$, the hedgehog of spininess $\tau$, is $\mathbb{I}(S)$, where $S$ is the set of ordinals $<\tau$.

In this section, we will make use of hedgehogs of the form $\mathbb{H}(S)$, where $\mathbb{H}$ denotes the Hilbert cube $\mathbb{I}^{\mathbb{N}}$. 
Lemma 1. Let $U_{1}$ be a uniformly open set of a uniform space $X, A$ any subset of $U_{1}$ and $U_{2}, U_{3}, \ldots$ uniformly open subsets of $U_{1}$. Then there exist a uniformly continuous $h: X \rightarrow \mathbb{H}$ and uniformly open sets $V_{1}, V_{2}, \ldots$ of $\mathbb{H}$ such that $U_{1}=\operatorname{coz} h, \operatorname{dim} h(A) \leq \operatorname{Dim} A$ and $h^{-1}\left(V_{i}\right)=U_{i}$ for $i=1,2, \ldots$.

Proof. Let $f=\triangle\left\{f_{i}: i \in \mathbb{N}\right\}: X \rightarrow \mathbb{I}^{\mathbb{N}}$, where each $f_{i}: X \rightarrow \mathbb{I}$ is uniformly continuous with coz $f_{i}=U_{i}$. The result now follows from Proposition 3 on taking $G_{1}=G_{2}=\cdots=U_{1}$. Note that the $h$ of Proposition 3 now maps $X$ into $\mathbb{I}^{\mathbb{N}} \times \mathbb{I}^{\mathbb{N}}$, and we may take $V_{i}$ to be the cozero set of the composite of the projection onto the first copy of $\mathbb{I}^{\mathbb{N}}$ followed by the projection onto the $i$ th copy of $\mathbb{I}$.

Lemma 2. Let $\left\{H_{s}: s \in S\right\}$ be a locally finite family consisting of mutually disjoint $\mathcal{U}$-open sets of a uniform space $(X, \mathcal{U})$. For each $s \in S$ let $A_{s}$ be a subset of $H_{s}$ with $\mathcal{U}-\operatorname{dim} A_{s} \leq n$. Let $A=\bigcup A_{s}$ and $H=\bigcup H_{s}$. Then there is a uniformity $\mathcal{V}$ finer than $\mathcal{U}$ on the topological space $X$ such that $\mathcal{V}-\operatorname{dim} A \leq n$ and $\mathcal{U}$ and $\mathcal{V}$ agree on $X \backslash H$ and also on each $H_{s}$.

Proof. By Lemma 1 , for each $s \in S$, there is a $\mathcal{U}$-uniformly continuous $h_{s}: X \rightarrow \mathbb{H}_{s}$ such that $H_{s}=\operatorname{coz} h_{s}$ and $\operatorname{dim} h_{s}\left(A_{s}\right) \leq n$. Define $h: X \rightarrow$ $\mathbb{H}(S)$ by $h(x)=h_{s}(x)$ if $x$ belongs to some (necessarily unique) $H_{s}$, and $h(x)=0$ if $x \notin H$. We note that $h$ is continuous: If $U$ is an open set of $\mathbb{H}(S)$ not containing 0 , then $h^{-1}(U)$ is clearly an open set of $H$, and if $F$ is a closed set of $\mathbb{H}(S)$ not containing 0 , then $h^{-1}(F)$ is closed as the union of the closed (in $X$ ) shrinking $\left\{h_{s}^{-1}\left(F \cap \mathbb{H}_{s}\right): s \in S\right\}$ of the locally finite collection $\left\{H_{s}: s \in S\right\}$.

We let $\mathcal{V}$ be the coarsest uniformity on $X$ that makes all maps $h$ as defined above uniformly continuous. As all such maps are $\mathcal{U}$-uniformly continuous on $X \backslash H$ as well as on each $H_{s}$, it follows that $\mathcal{U}$ and $\mathcal{V}$ agree on these sets. It remains to prove $\mathcal{V}-\operatorname{dim} A \leq n$.

Consider a $\mathcal{V}$-open cover $\left\{U_{i}: i=1, \ldots, k\right\}$ of $A$. As $A_{s} \cap U_{i}$ is a $\mathcal{U}$ open set of $A_{s}$, it is the trace on $A_{s}$ of some $\mathcal{U}$-open set $V_{s i}$ of $H_{s}$. By Lemma 1, we may assume in the first paragraph of the present proof that there are open sets $W_{s i}$ of $\mathbb{H}_{s}$ with $h_{s}^{-1}\left(W_{s i}\right)=V_{s i}$. Note that for each $s \in S, h_{s}\left(A_{s}\right) \subset \mathbb{H}_{s} \backslash\{0\}$ and therefore $B=\bigcup h_{s}\left(A_{s}\right)$ is the topological sum of $h_{s}\left(A_{s}\right), s \in S$. Consequently, $\operatorname{dim} B \leq n$. Let $\left\{G_{i}: i=1, \ldots, k\right\}$ be an open cover of $B$ of order $\leq n$ refining $\left\{B \cap \bigcup_{s \in S} W_{s i}: i=1, \ldots, k\right\}$. Then $\left\{h^{-1}\left(G_{i}\right): i=1, \ldots, k\right\}$ is a $\mathcal{V}$-open cover of $A$ of order $\leq n$ refining the $\mathcal{V}$-open cover $\left\{U_{i}: i=1, \ldots, k\right\}$ of $A$. Hence $\mathcal{V}$-dim $A \leq n$.

Let $\mathbb{F}(S)$ denote the subset of $\prod_{s \in S} \mathbb{I}_{s}$ consisting of all points $x=\left(x_{s}\right)$ that have only a finite number of non-zero coordinates $x_{s}$. It will be understood to be equipped with the metric $d$ defined by $d(x, y)=\sup _{s \in S}\left|x_{s}-y_{s}\right|$. It is important to note that for every finite subset $T$ of $S$, the subspace $\left\{x: x_{s}=0\right.$ for $\left.s \notin T\right\}$ of $\mathbb{F}(S)$ is homeomorphic to $\prod_{s \in T} \mathbb{I}_{s}$. 
A family of maps $\left\{f_{s}: X \rightarrow \mathbb{I}_{s}: s \in S\right\}$, where $\mathbb{I}_{s}$ is a copy of the unit interval, will be called locally finite (resp. point-finite) if the collection $\left.\left\{\operatorname{coz} f_{s}: s \in S\right)\right\}$ is locally finite (resp. point-finite) in $X$. A point-finite family $\left\{f_{s}: X \rightarrow \mathbb{I}_{s}: s \in S\right\}$ induces a function $f=\triangle_{s \in S} f_{s}: X \rightarrow \mathbb{F}(S)$, which is clearly continuous if the family of maps is locally finite.

TheOREm 1. Let $\left\{f_{s}: s \in S\right\}$ be a locally finite family of uniformly continuous maps on a uniform space $(X, \mathcal{U})$ into the unit interval such that $\mathcal{U}$-dim $G_{s} \leq n$ for each $s \in S$, where $G_{s}=\operatorname{coz} f_{s}$. Let $f: X \rightarrow \mathbb{F}(S)$ be the map induced by the family $\left\{f_{s}: s \in S\right\}$ and $G=\bigcup_{s \in S} G_{s}$. Then there is a uniformity $\mathcal{V}$ on $X$ finer than $\mathcal{U}$ which agrees with $\mathcal{U}$ on $X \backslash G$, makes $f$ uniformly continuous and satisfies $\mathcal{V}-\operatorname{dim} G \leq n$.

Proof. To begin with, we let $\mathcal{U}_{0}$ be the coarsest uniformity on $X$ bigger than $\mathcal{U}$ that makes $f$ uniformly continuous. Let $E_{0}=\emptyset$, and for each $n \in \mathbb{N}$, let $E_{n}$ consist of those points $x$ of $G$ for which the set $\left\{s \in S: x \in G_{s}\right\}$ has at most $n$ elements. Let $H_{s}=\left\{x \in \mathbb{F}(S): x_{s}>0\right\} \cap f(X)$ and $H=\bigcup_{s \in S} H_{s}$. Clearly, $f^{-1}\left(H_{s}\right)=G_{s},\left\{H_{s}: s \in S\right\}$ is a point-finite family consisting of open sets of $f(X)$, and for each $n \in \mathbb{N}$, the set $F_{n}$ consisting of those points $x$ of $H$ for which $\left\{s \in S: x \in H_{s}\right\}$ has at most $n$ elements is closed in $H$. Hence $G=f^{-1}(H)$ is $\mathcal{U}_{0}$-open and each $E_{n}=f^{-1}\left(F_{n}\right)$ is $\mathcal{U}_{0}$-closed in $G$.

Note that if $A$ is a subset of $X$ such that $\left\{s \in S:\left.f_{s}\right|_{A} \neq 0\right\}$ is finite, then $\left.f\right|_{A}$ is $\mathcal{U}$-uniformly continuous and consequently $\left.\mathcal{U}_{0}\right|_{A}=\left.\mathcal{U}\right|_{A}$. Therefore, by the subset theorem, $\mathcal{U}_{0}-\operatorname{dim} A=\mathcal{U}-\operatorname{dim} A \leq n$ for any subset $A$ of $X$ of the form $\left(E_{m} \backslash E_{m-1}\right) \cap \bigcap_{s \in M} G_{s}$, for any $m \in \mathbb{N}$ and any subset $M$ of $S$ with $|M|=m$.

We construct by induction for each $k \in \mathbb{N}$ a uniformity $\mathcal{U}_{k}$ on $X$ which is finer than $\mathcal{U}_{k-1}$, agrees with it on the set $(X \backslash G) \cup E_{k-1}$ and satisfies $\mathcal{U}_{k}-\operatorname{dim}\left(E_{k} \backslash E_{k-1}\right) \leq n$ and $\mathcal{U}_{k}-\operatorname{dim} A \leq n$ for any subset $A$ of $X$ of the form $\left(E_{m} \backslash E_{m-1}\right) \cap \bigcap_{s \in M} G_{s}$, for any $m>k$ and any subset $M$ of $S$ with $|M|=m$. Assume $\mathcal{U}_{k-1}$ has been constructed as required. It suffices to construct $\mathcal{U}_{k}$ with the properties just stated.

We observe that $\left\{\left(F_{k} \backslash F_{k-1}\right) \cap \bigcap_{s \in K} H_{s}: K \subset S,|K|=k\right\}$ is a discrete (in the metric space $H \backslash F_{k-1}$ ) clopen cover of $F_{k} \backslash F_{k-1}$. Consequently, there exist mutually disjoint open sets $V_{K}$ of $H$ such that $\left(F_{k} \backslash F_{k-1}\right) \cap$ $\bigcap_{s \in K} H_{s} \subset V_{K} \subset \bigcap_{s \in K} H_{s}$. Let $U_{K}=f^{-1}\left(V_{K}\right)$ and $U=\bigcup\left\{U_{K}: K \subset S\right.$, $|K|=k\}$. Then $A_{K}=\left(E_{k} \backslash E_{k-1}\right) \cap \bigcap_{s \in K} G_{s} \subset U_{K} \subset \bigcap_{s \in K} G_{s}$ and $\left\{U_{K}: K \subset S,|K|=k\right\}$ is a locally finite collection of $X$ consisting of mutually disjoint $\mathcal{U}_{k-1}$-open sets of $G \backslash E_{k-1}$. Also, by the induction hypothesis, $\mathcal{U}_{k-1}$-dim $A_{K} \leq n$. We can therefore apply Lemma 2 to construct a uniformity $\mathcal{U}_{k}$ on $X$ finer than $\mathcal{U}_{k-1}$ and agreeing with $\mathcal{U}_{k-1}$ on each $U_{K}$ as well as on $X \backslash U$, which contains $(X \backslash G) \cup E_{k-1}$, and such that $\mathcal{U}_{k}-\operatorname{dim}\left(E_{k} \backslash E_{k-1}\right) \leq$ $n$. Finally, consider a subset $A$ of $X$ of the form $\left(E_{m} \backslash E_{m-1}\right) \cap \bigcap_{s \in M} G_{s}$, 
where $m>k$ and $M$ is a subset of $S$ with $|M|=m$. Note that $\mathcal{U}_{k-1}$ and $\mathcal{U}_{k}$ agree on $A \backslash U$ as well as on each $A \cap U_{K}$, and $A \cap U_{K} \neq \emptyset$ implies $K \subset M$. Thus, $A \cap U_{K} \neq \emptyset$ for only finitely many $K$ 's. By Proposition 1 and the induction hypothesis, $\mathcal{U}_{k}-\operatorname{dim}(A \backslash U)=\mathcal{U}_{k-1}-\operatorname{dim}(A \backslash U) \leq n$ and $\mathcal{U}_{k}$ - $\operatorname{dim}\left(A \cap U_{K}\right)=\mathcal{U}_{k-1}-\operatorname{dim}\left(A \cap U_{K}\right) \leq n$. Now, Corollary 1 implies that $\mathcal{U}_{k}$-dim $A \leq n$, which completes the construction.

To complete the proof, we let $\mathcal{V}$ be the uniformity on $X$ generated by $\mathcal{U}_{0}, \mathcal{U}_{1}, \mathcal{U}_{2}, \ldots$ Evidently, $\mathcal{V}$ and $\mathcal{U}_{k}$ agree on $E_{k}$. Hence $\mathcal{V}$-dim $\left(E_{k} \backslash E_{k-1}\right)$ $\leq n$. Finally, by Corollary $2, \mathcal{V}-\operatorname{dim} G \leq n$.

4. Topologies generated by families of maps. Recall that the topology of a space $X$ is said to be generated by a family of maps $\left\{f_{s}: X \rightarrow Y_{s}\right.$ : $s \in S\}$ if it is the coarsest topology on $X$ that makes every $f_{s}$ continuous. This simply means that $\left\{f_{s}^{-1}(G): G\right.$ open in $\left.Y_{s}, s \in S\right\}$ is a subbase for the topology of $X$.

TheOREM 2. A $T_{1}$ space $X$ is metrizable iff the topology of $X$ is generated by a $\sigma$-locally finite collection of maps into $\mathbb{I}$.

Proof. Suppose first that $X$ is metrizable. Let $\left\{G_{s}: s \in S_{i}, i \in \mathbb{N}\right\}$ be a $\sigma$-locally finite base for the topology of $X$. Pick $f_{s}: X \rightarrow \mathbb{I}$ with coz $f_{s}=G_{s}$ for each $s \in S_{i}$ and each $i \in \mathbb{N}$. Then $\left\{f_{s}: s \in S_{i}, i \in \mathbb{N}\right\}$ is a $\sigma$-locally finite collection of maps into $\mathbb{I}$ that generates the topology of $X$.

Conversely, let $\left\{f_{s}: s \in S_{i}, i \in \mathbb{N}\right\}$ be a $\sigma$-locally finite collection of maps into $\mathbb{I}$ that generates the topology of $X$. For each $i \in \mathbb{N}$, let $f_{i}=\triangle_{s \in S_{i}} f_{s}$ : $X \rightarrow \mathbb{F}\left(S_{i}\right)$ be the map induced by the locally finite collection $\left\{f_{s}: s \in S_{i}\right\}$. Each $f_{i}$ is continuous and so is $f=\triangle_{i \in \mathbb{N}} f_{i}: X \rightarrow \prod_{i \in \mathbb{N}} \mathbb{F}\left(S_{i}\right)$. First note that $f$ is injective. For if $f(x)=f(y)$, then $f_{s}(x)=f_{s}(y)$ for all $s \in \bigcup_{i} S_{i}$, which implies that $\{x\}$ and $\{y\}$ have the same closure and therefore $x=y$. Also, a set of the form $f_{s}^{-1}(G)$, where $G$ is open in $\mathbb{I}$ and $s \in S_{i}$, is the inverse image under $f$ of the open set $p_{i}^{-1}\left(p_{s}^{-1}(G)\right)$ of $\prod_{i \in \mathbb{N}} \mathbb{F}\left(S_{i}\right)$, where $p_{i}: \prod_{i \in \mathbb{N}} \mathbb{F}\left(S_{i}\right) \rightarrow \mathbb{F}\left(S_{i}\right)$ and $p_{s}: \mathbb{F}\left(S_{i}\right) \rightarrow \mathbb{I}_{s}$ are the obvious projections. As these sets generate the topology of $X$, it follows that $f$ is an embedding of $X$ into a metrizable space. Hence $X$ is metrizable.

The proof of the following result is essentially the same as the proof of $(\mathrm{c}) \Leftrightarrow(\mathrm{d})$ of Theorem 1.4 in [10], giving four characterizations of Eberlein compact spaces. Condition (d) is Rosenthal's characterization in terms of $\sigma$-point-finite, separating families of cozero sets [12].

Theorem 3. A $T_{1}$ space $X$ can be embedded in some $c_{0}(S)$ iff the topology of $X$ is generated by a $\sigma$-point-finite collection of maps into $\mathbb{I}$.

Proof. For $s \in S$ and $n \in \mathbb{N}$, let $f_{s, n}: c_{0}(S) \rightarrow \mathbb{I}$ be the composite of the projection $c_{0}(S) \rightarrow \mathbb{I}_{s}$ followed by the map that sends $x$ to 0 or 
$((n+1) x-1)) / n$ depending on whether $x \leq 1 /(n+1)$ or $x>1 /(n+1)$. Then for each $n,\left\{f_{s, n}: s \in S\right\}$ is point-finite and one readily sees that $\left\{f_{s, n}: s \in S, n \in \mathbb{N}\right\}$ generates the topology of $c_{0}(S)$. Consequently, the topology of any subspace $X$ of $c_{0}(S)$ is generated by the $\sigma$-point-finite family $\left\{\left.f_{s, n}\right|_{X}: s \in S, n \in \mathbb{N}\right\}$.

Conversely, suppose $\left\{f_{s}: X \rightarrow \mathbb{I}_{s}: s \in S_{i}, i \in \mathbb{N}\right\}$ is a $\sigma$-point-finite collection of maps into the unit interval that generates the topology of $X$. Let $S=\bigcup_{i \in \mathbb{N}} S_{i}$. Then $f=\triangle_{s \in S} f_{s}: X \rightarrow \prod_{s \in S} \mathbb{I}_{s}$ is an embedding. For each $n \in \mathbb{N}$ and $s \in S_{n}$, we can of course assume that $f_{s}$ maps into $[0,1 / n]$. Then $f_{s}(x) \geq 1 / n$ implies $s \in S_{1} \cup \cdots \cup S_{n}$, which makes $f$ an embedding of $X$ into $c_{0}(S)$.

One can readily deduce from Theorem 3 the well known result that the product of countably many Eberlein compact spaces is Eberlein compact.

For our final result in this section, we will need the following lemma from [14].

Lemma 3. Every point-finite family of open sets of a Baire space $X$ is locally finite on a dense open subset of $X$.

TheOREM 4. A Baire subspace $Y$ of $c_{0}(S)$ contains a dense $G_{\delta}$ metrizable subspace $X$ with $\operatorname{dim} X \leq \operatorname{dim} Y$.

Proof. Theorem 3 yields a $\sigma$-point-finite family $\left\{g_{s}: Y \rightarrow \mathbb{I}_{s}: s \in S_{i}\right.$, $i \in \mathbb{N}\}$ of maps into the unit interval that generates the topology of $Y$. For each $i \in \mathbb{N},\left\{\operatorname{coz} g_{s}: s \in S_{i}\right\}$ is point-finite. By Lemma $3,\left\{\operatorname{coz} g_{s}: s \in S_{i}\right\}$ is locally finite on a dense open subset $X_{i}$ of $Y$. Let $X=\bigcap_{i \in \mathbb{N}} X_{i}$, and for each $s \in S=\bigcup_{i \in \mathbb{N}} S_{i}$, let $f_{s}=\left.g_{s}\right|_{X}$ and $G_{s}=\operatorname{coz} f_{s}$. Then $X$ is a dense $G_{\delta}$ of the Baire space $Y$, and evidently, $\left\{f_{s}: X \rightarrow \mathbb{I}_{s}: s \in S_{i}, i \in \mathbb{N}\right\}$ is a $\sigma$-locally finite collection of maps into the unit interval generating the topology of $X$. By Theorem 2, $X$ is metrizable.

Let $\mathcal{U}_{0}$ denote the largest uniformity on $Y$. Then each $G_{s}$ is an $\mathcal{U}_{0}$-open set of $X$, and by the subset theorem, $\mathcal{U}_{0}-\operatorname{dim} G_{s} \leq \mathcal{U}_{0}$ - $\operatorname{dim} Y=\operatorname{dim} Y$. For each $i \in \mathbb{N}$, let $f_{i}=\triangle_{s \in S_{i}} f_{s}: X \rightarrow \mathbb{F}\left(S_{i}\right)$. Clearly, we can assume that for each $i \in \mathbb{N}$, there is $s \in S_{i}$ with $G_{s}=X$ so that $\bigcup_{s \in S_{i}} G_{s}=X$. Applying Theorem 1, we can construct uniformities $\mathcal{U}_{0} \subset \mathcal{U}_{1} \subset \mathcal{U}_{2} \subset \cdots$ such that $\mathcal{U}_{i}$ makes $f_{i}$ uniformly continuous and $\mathcal{U}_{i}$-dim $X \leq n$. Let $\mathcal{U}=\bigcup_{i \in \mathbb{N}} \mathcal{U}_{i}$. Then $(X, \mathcal{U})$ is the inverse limit of the sequence $\left(X, \mathcal{U}_{i}, p_{i, j}\right)$, where each bonding map is the identity function on $X$. Hence, by Proposition $4, \mathcal{U}$ - $\operatorname{dim} X \leq n$. Moreover, $f=\triangle_{i \in \mathbb{N}} f_{i}: X \rightarrow \prod_{i \in \mathbb{N}} \mathbb{F}\left(S_{i}\right)$ is $\mathcal{U}$-uniformly continuous in addition to being, as in Theorem 2, a topological embedding. Consider an open set $G$ of $X$. Because $f$ is an embedding, there is an open set $H$ of $\prod_{i \in \mathbb{N}} \mathbb{F}\left(S_{i}\right)$ with $G=f^{-1}(H)$. As $f$ is $\mathcal{U}$-uniformly continuous, $G$ and thus every open set of $X$ is $\mathcal{U}$-open. Hence $\operatorname{dim} X=\mathcal{U}$-dim $X \leq n$. 
Example 1 at the end of Section 5 shows that in Theorem 4 we do not always have $\operatorname{dim} X=\operatorname{dim} Y$.

\section{The inductive dimension of the Kimura-Morishita compact-} ification. In this section, we shall need various properties of the inductive dimensions $\mathcal{U}$-ind $X$ and $\mathcal{U}$-Ind $X$ of a uniform space $(X, \mathcal{U})$, which were studied in [3], where proofs of all results that we quote can be found. Their definitions are obtained by respectively replacing open sets and closed sets by uniformly open sets and uniformly closed sets in the corresponding definitions of the inductive dimension ind and Ind of a topological space. Thus, $\mathcal{U}$-Ind $X=-1$ (resp. $\mathcal{U}$-ind $X=-1$ ) iff $X=\emptyset$, and, for $n=0,1, \ldots$, $\mathcal{U}$-Ind $X \leq n$ (resp. $\mathcal{U}$-ind $X \leq n$ ) iff any disjoint uniformly closed sets $F_{1}, F_{2}$ of $X$ (resp. one of which is a singleton) are respectively contained in disjoint uniformly open sets $G_{1}, G_{2}$ of $X$ with $\mathcal{U}$ - $\operatorname{Ind}\left(X \backslash\left(G_{1} \cup G_{2}\right)\right) \leq n-1$ $\left(\right.$ resp. $\mathcal{U}$-ind $\left.\left(X \backslash\left(G_{1} \cup G_{2}\right)\right) \leq n-1\right)$.

Both these functions satisfy the subset theorem just as $\mathcal{U}$-dim. In addition, $\mathcal{U}$-Ind satisfies a countable sum theorem analogous to Proposition 2. Also, ind $X \leq \mathcal{U}$-ind $X \leq \mathcal{U}$-Ind $X$, Ind $X \leq \mathcal{U}$-Ind $X$, and if $X$ is Lindelöf, then $\mathcal{U}$-ind $X=\mathcal{U}$-Ind $X$. We will additionally need the following version of the Urysohn inequality for $\mathcal{U}$-Ind, which is slightly stronger than Proposition 10 of [3].

Proposition 5. Let $A$ be a subspace of a uniform space $(X, \mathcal{U})$ such that $\mathcal{U}$-Ind $A \leq m$ and $\mathcal{U}$-Ind $B \leq n$ for every $\mathcal{U}$-closed subset $B$ of $X$ disjoint from $A$. Then $\mathcal{U}$-Ind $X \leq m+n+1$.

Sketch of proof. Let $F_{1}, F_{2}$ be disjoint uniformly closed subsets of $X$. As in the proof of Proposition 10 of [3], $F_{1}, F_{2}$ are respectively contained in disjoint uniformly open subsets $G_{1}, G_{2}$ of $X$ with $\mathcal{U}$ - $\operatorname{Ind}\left(A \backslash\left(G_{1} \cup G_{2}\right)\right) \leq$ $m-1$. By the obvious induction hypothesis, $\mathcal{U}$-Ind $\left(X \backslash\left(G_{1} \cup G_{2}\right)\right) \leq(m-1)$ $+n+1=m+n$. Hence $\mathcal{U}$-Ind $X \leq m+n+1$.

$J(\tau)$, the hedgehog of spininess $\tau$, has an Eberlein compactification $J^{*}(\tau)$ defined as follows (cf. [9]). With the notation established in the opening paragraph of Section 3, the underlying set of $J^{*}(\tau)$ is $\mathbb{I}(S)$, where $S$ is the set of ordinals $\leq \tau$. Let $p: \mathbb{I}(S) \rightarrow \mathbb{I}$ be the function that sends 0 to 0 and $(x, s)$ to $x$. Also, for $s<\tau$, let $p_{s}: \mathbb{I}(S) \rightarrow \mathbb{I}$ send $(x, s)$ to $x$ and everything else to 0 . The basic neighbourhoods of the various points of $J^{*}(\tau)$ are defined to be the following: for 0 , all sets of the form $p^{-1}([0, a)), 0<a<1$; for points of the form $(x, s)$, where $s<\tau$, all sets of the form $p_{s}^{-1}(U)$, where $U$ is an open interval of $\mathbb{I}$ containing $x$; finally, for points of the form $(x, \tau)$, all sets of the form $p^{-1}(U) \backslash \bigcup_{s \in F} \mathbb{I}_{s}$, where $U$ is an open interval of $\mathbb{I}$ containing $x$ and $F$ is a finite set of ordinals $<\tau$. 
One readily checks that $\left(J^{*}(\tau) \backslash \mathbb{I}_{\tau}\right) \cup\{0\}$ is homeomorphic to $J(\tau)$ and $J^{*}(\tau)$ is compact Hausdorff. It is Eberlein compact because the topology of $J^{*}(\tau)$ is generated by the point-finite family of maps $\{p\} \cup\left\{p_{s}: s<\tau\right\}$.

Observe that all basic neighbourhoods defined above are cozero sets and are therefore uniformly open with respect to the unique uniformity on $J^{*}(\tau)$, and their closures are zero sets and are therefore uniformly closed.

We will be working inside the Eberlein compact space $J^{*}(\tau)^{\mathbb{N}}$. Its unique uniformity will be denoted by $\mathcal{U}$. We use $\mathbb{Q}$ and $\mathbb{P}$ respectively for the sets of rationals and of irrationals in $(0,1]$. For each non-negative integer $n$, let

$$
\begin{aligned}
& K_{n}^{*}(\tau)=\left\{z=\left(z_{i}\right) \in J^{*}(\tau)^{\mathbb{N}}:\left|\left\{i: p\left(z_{i}\right) \in \mathbb{Q}\right\}\right| \leq n\right\}, \\
& M_{n}^{*}(\tau)=\left\{z \in J^{*}(\tau)^{\mathbb{N}}:\left|\left\{i: p\left(z_{i}\right) \in \mathbb{Q}\right\}\right|=n\right\} .
\end{aligned}
$$

Kimura and Morishita [9] have proved that a compact subspace of $K_{n}^{*}(\tau)$ has $\operatorname{dim} \leq n$, and every metrizable space $M$ with $\operatorname{dim} M=n$ and $w M=\tau$ has a compactification $E$ inside $K_{n}^{*}(\tau)$ with $\operatorname{dim} E=n$. We claim that in fact Ind $E=n$. This will follow from Theorem 5 , where we prove that every Lindelöf subspace of $K_{n}^{*}(\tau)$ has Ind $\leq n$.

For any subset $J$ of $\mathbb{N}$ with $|J|=n$, let

$$
M_{n}^{*}(\tau, J)=\left\{z \in M_{n}^{*}(\tau): p\left(z_{i}\right) \in \mathbb{Q} \text { for } i \in J\right\} .
$$

Lemma 4. For any $J \subset \mathbb{N}$ with $|J|=n, \mathcal{U}$-ind $M_{n}^{*}(\tau, J)=0$.

Proof. Let $V$ be an open neighbourhood of a point $z$ in $J^{*}(\tau)^{\mathbb{N}}$. Consider a neighbourhood $U=\bigcap_{i \in L} \pi_{i}^{-1}\left(U_{i}\right)$ of $z$ with closure inside $V$, where $L$ is a finite subset of $\mathbb{N}$ containing $J, \pi_{i}$ is the $i$ th projection from $J^{*}(\tau)^{\mathbb{N}}$ to $J^{*}(\tau)$, and $U_{i}$ is a basic neighbourhood of $z_{i}$ chosen so that $p\left(\operatorname{Bd} U_{i}\right)$ is a subset of $\mathbb{P}$ if $i \in J$, and a subset of $\mathbb{Q}$ if $i \in L \backslash J$. Then in $J^{*}(\tau)^{\mathbb{N}}$, $U$ is uniformly open, its closure is uniformly closed, and one can verify that $\operatorname{Bd} U \cap\left(M_{n}^{*}(\tau, J)\right)=\emptyset$. This clearly implies the result.

Theorem 5. For any Lindelöf subspace $X$ of $K_{n}^{*}(\tau), \mathcal{U}$-Ind $X \leq n$ and therefore Ind $X \leq n$.

Proof. $M_{n}^{*}(\tau)$ is the union of subspaces $Z_{1}, Z_{2}, \ldots$ of the form $\{z \in$ $M_{n}^{*}(\tau): p\left(z_{i}\right)=q_{i}$ for $\left.i \in J\right\}$, where $J$ is a subset of $\mathbb{N}$ with $|J|=n$ and $q_{i}$ is a fixed element of $\mathbb{Q}$ for each $i \in J$. For each $i$, by Lemma 4 and the subset theorem, $\mathcal{U}$-ind $\left(X \cap Z_{i}\right) \leq 0$. Also, $Z_{i}$ is uniformly closed in $K_{n}^{*}(\tau)$. Hence, $X \cap Z_{i}$ is Lindelöf and therefore $\mathcal{U}$ - $\operatorname{Ind}\left(X \cap Z_{i}\right) \leq 0$. Now, by the countable sum theorem for $\mathcal{U}$-Ind, we have $\mathcal{U}$-Ind $\left(X \cap M_{n}^{*}(\tau)\right) \leq 0$.

In particular, Theorem 5 is valid for $n=0$. Note that for $k>0, K_{n}^{*}(\tau)$ is the disjoint union of $K_{n-1}^{*}(\tau)$ and $M_{n}^{*}(\tau)$. Assume that $\mathcal{U}$-Ind $Y \leq n-1$ for any Lindelöf subspace $Y$ of $K_{n-1}^{*}(\tau)$. Then any uniformly closed subset $B$ of the Lindelöf space $X$ disjoint from $A=X \cap M_{n}^{*}(\tau)$ will be a Lindelöf 
subspace of $K_{n-1}^{*}(\tau)$ and hence $\mathcal{U}$-Ind $B \leq n-1$. Now, by Proposition 5 , $\mathcal{U}$-Ind $X \leq 0+(n-1)+1=n$, which completes the proof.

We conclude this section with an example that complements Theorem 4.

EXAMPLE 1. Let $\tau$ be the cardinality of the continuum and write $\mathbb{I} \backslash\{0\}=\left\{x_{s}: s<\tau\right\}$, where each point occurs continuum many times. Let $Y$ be the Eberlein compact subspace of $J^{*}(\tau)$ consisting of $I_{\tau}$ together with all points $\left(x_{s}, s\right), s<\tau$. The set $X$ of $G_{\delta}$ points of $Y$ consists of the points $\left(x_{s}, s\right), s<\tau$, together with 0. Clearly, $X$ is the largest metrizable $G_{\delta}$ subspace of $Y$, while $\operatorname{dim} X=0<\operatorname{dim} Y=1$.

6. Eberlein compactifications and the subset theorem. Let $X$ be a subspace of $Y$. Then $X$ is a $d$-regular subspace of $Y$, or $Y$ is a $d$ regular extension of $X$, if every cozero set of a $X$ is the union of a $\sigma$-locally finite (in $X$ ) collection consisting of clopen subsets of traces on $X$ of cozero sets of $Y$. A very general subset theorem due to Pasynkov [11] asserts that $\operatorname{dim} X \leq \operatorname{dim} Y$ if $X$ is a $d$-regular subspace of $Y$.

The Kimura-Morishita [9] compactifications turn out to be $d$-regular in a very strong manner.

TheOREM 6. Let $\left\{G_{i, s}: i \in \mathbb{N}, s \in S\right\}$ be a $\sigma$-discrete base of a metrizable space $M$ with $\operatorname{dim} M=n$ and $w M=\tau$, and set $G_{i}=\bigcup\left\{G_{i, s}: s \in S\right\}$. Then there is an embedding $h: M \rightarrow J(\tau)^{\mathbb{N}}$ such that $E=\operatorname{cl}(h(M)) \subset$ $K_{n}^{*}(\tau)$, where cl denotes closure in $J^{*}(\tau)^{\mathbb{N}}$, and $E$ contains cozero sets $H_{i}$ with $G_{i}=h^{-1}\left(H_{i}\right)$. In fact the set of all such maps is residual in the complete metric space $C\left(M, J(\tau)^{\mathbb{N}}\right)$ of all maps of $M$ into $J(\tau)^{\mathbb{N}}$.

Proof. For each $i, G_{i}=\bigcup\left\{F_{i, j}: j \in \mathbb{N}\right\}$, where each $F_{i, j}$ is closed in $M$. By the work of Kimura and Morishita [9], the set of all embeddings $h: M \rightarrow$ $J(\tau)^{\mathbb{N}}$ with $E=\operatorname{cl}(h(M)) \subset K_{n}^{*}(\tau)$ and $\operatorname{cl}\left(h\left(F_{i, j}\right)\right) \cap \operatorname{cl}\left(h\left(M \backslash G_{i}\right)\right)=\emptyset$ for all $i, j \in \mathbb{N}$ is residual in $C\left(M, J(\tau)^{\mathbb{N}}\right)$. For any one such $h$, pick a cozero set $H_{i, j}$ of $E$ containing $\operatorname{cl}\left(h\left(F_{i, j}\right)\right)$ and disjoint from $\operatorname{cl}\left(h\left(M \backslash G_{i}\right)\right)$. Finally, set $H_{i}=\bigcup\left\{H_{i, j}: j \in \mathbb{N}\right\}$.

Call a subspace $X$ of $Y$ countably accessible if for every cozero set $G$ of $X$ there are maps $f_{i, s}: X \rightarrow \mathbb{I}, i \in \mathbb{N}, s \in S$, such that each $f_{i, s} \mid \operatorname{coz} f_{i, s}$ has a continuous extension from $Y$ to $\mathbb{I},\left\{\operatorname{coz} f_{i, s}: s \in S\right\}$ is locally finite in $X$ for each $i$, and $G$ is open in the topology on $X$ generated by $\left\{f_{i, s}: i \in \mathbb{N}, s \in S\right\}$.

Evidently, the metrizable subspace in Theorem 4 is a countably accessible subspace of the Baire subspace of $c_{0}(S)$. Given a map $g: Y \rightarrow \mathbb{I}$ and a clopen subset $U$ of $X \cap \operatorname{coz} g$, define $f: X \rightarrow \mathbb{I}$ by $f(x)=g(x)$ or 0 depending on whether $x$ belongs to $U$ or not. Then $f$ is continuous with coz $f=U$. Hence, a $d$-regular subspace $X$ of $Y$ is countably accessible. 
We conclude this paper with the following subset theorem that generalizes Pasynkov's theorem [11] and also covers the case of the metrizable subspace in Theorem 4.

TheOREM 7. Let $X$ be a countably accessible subspace of $Y$. Then $\operatorname{dim} X$ $\leq \operatorname{dim} Y$.

Proof. Let $\mathcal{G}$ be a finite cozero cover of $X$ and suppose $\operatorname{dim} Y=n$. Let $\mathcal{U}_{-1}$ be the biggest uniformity on $Y$. Then $\mathcal{U}_{-1}$-dim $X \leq \mathcal{U}_{-1}$-dim $Y=n$.

Consider the set $\mathcal{S}$ of all uniformities $\mathcal{W}$ on $X$ finer than $\mathcal{U}_{-1}$ and such that $\mathcal{W}$-dim $X \leq n$. Order $\mathcal{S}$ by inclusion. Then Proposition 4 readily implies that the union of any chain of elements in $\mathcal{S}$ is again an element of $\mathcal{S}$. Hence there is a maximal element $\mathcal{U}_{0}$ in $\mathcal{S}$.

Now suppose $U=\operatorname{coz} h$ for some map $h: X \rightarrow \mathbb{I}$ such that $h \mid U$ has a continuous extension from $Y$ to $\mathbb{I}$. This implies that $h$ is $\mathcal{U}_{0}$-uniformly continuous on both $U$ and $X \backslash U$. Let $\mathcal{V}$ be the uniformity on $X$ finer than $\mathcal{U}_{0}$ generated by all maps $g: X \rightarrow \mathbb{I}$ such that each $g$ is $\mathcal{U}_{0}$-uniformly continuous on both $U$ and $X \backslash U$. Then $\mathcal{V}$ agrees with $\mathcal{U}_{0}$ on both $U$ and $X \backslash U$, and $U$ is $\mathcal{V}$-open. Consequently, by the subset theorem, $\mathcal{V}$-dim $U \leq n$ and $\mathcal{V}$-dim $(X \backslash U) \leq n$. Therefore, by Corollary $1, \mathcal{V}$-dim $X \leq n$. This means that $\mathcal{V}=\mathcal{U}_{0}$ and $h$ is $\mathcal{U}_{0}$-uniformly continuous.

As $X$ is a countably accessible subspace of $Y$, there are maps $f_{i, s}: X \rightarrow \mathbb{I}$, $i \in \mathbb{N}, s \in S$, such that each $f_{i, s} \mid \operatorname{coz} f_{i, s}$ has a continuous extension from $Y$ to $\mathbb{I},\left\{\operatorname{coz} f_{i, s}: s \in S\right\}$ is locally finite in $X$ for each $i$, and every member of $\mathcal{G}$ is open in the topology on $X$ generated by $\left\{f_{i, s}: i \in \mathbb{N}, s \in S\right\}$. Note that, by the preceding paragraph, each $f_{i, s}$ is $\mathcal{U}_{0}$-uniformly continuous, and for each $i$, we can assume $\operatorname{coz} f_{i, s}=X$ for some $s$. For each $i$, let $f_{i}=\triangle_{s \in S} f_{i, s}$ : $X \rightarrow \mathbb{F}(S)$. Applying Theorem 1 , we can construct a uniformity $\mathcal{U}_{i}$ finer than $\mathcal{U}_{0}$ such that $\mathcal{U}_{i}$ makes $f_{i}$ uniformly continuous and $\mathcal{U}_{i}$-dim $X \leq n$. Then, by the definition of $\mathcal{U}_{0}, \mathcal{U}_{i}=\mathcal{U}_{0}$. Thus, each $f_{i}$ and therefore $f=\triangle_{i \in \mathbb{N}} f_{i}$ : $X \rightarrow \mathbb{F}(S)^{\mathbb{N}}$ is $\mathcal{U}_{0}$-uniformly continuous. As each $f_{i, s}$ is the composite of the $i$ th projection $\mathbb{F}(S)^{\mathbb{N}} \rightarrow \mathbb{F}(S)$ followed by the sth projection $\mathbb{F}(S) \rightarrow \mathbb{I}$, both uniformly continuous, one readily sees that $\mathcal{G}$ is a subset of the topology $\left\{f^{-1}(G): G\right.$ open in $\left.\mathbb{F}(S)^{\mathbb{N}}\right\}$ on $X$. Consequently, $\mathcal{G}$ consists of $\mathcal{U}_{0^{-}}$open sets and therefore has a finite $\mathcal{U}_{0}$-open refinement of order $\leq \mathcal{U}_{0}$-dim $X \leq n$. Thus, $\operatorname{dim} X \leq n=\operatorname{dim} Y$.

\section{References}

[1] D. Amir and J. Lindenstrauss, The structure of weakly compact sets in Banach spaces, Ann. of Math. (2) 88 (1968), 35-46.

[2] M. G. Charalambous, A new covering dimension function for uniform spaces, J. London Math. Soc. (2) 11 (1975), 137-143. 
[3] M. G. Charalambous, Inductive dimension theory for uniform spaces, Ann. Univ. Sci. Budapest. Sect. Math. 17 (1974), 21-28.

[4] - , The dimension of inverse limits, Proc. Amer. Math. Soc. 58 (1976), 289-295.

[5] - Further theory and applications of covering dimension of uniform spaces, Czechoslovak Math. J. 41 (116) (1991), 378-394.

[6] G. Dimov, Baire subspaces of $c_{0}(\Gamma)$ have dense $G_{\delta}$ metrizable subsets, Rend. Circ. Mat. Palermo (2) Suppl. 18 (1988), 275-285.

[7] R. Engelking, General Topology, Heldermann, Lemgo, 1995.

[8] —, Theory of Dimensions, Finite and Infinite, Heldermann, Berlin, 1989.

[9] T. Kimura and K. Morishita, On Eberlein compactifications of metrizable spaces, Fund. Math. 171 (2002), 223-234.

[10] E. Michael and M. E. Rudin, A note on Eberlein compacts, Pacific J. Math. 72 (1977), 487-495.

[11] B. A. Pasynkov, On the monotonicity of dimension, Soviet Math. Dokl. 26 (1982), 654-658.

[12] H. P. Rosenthal, The heredity problem for weakly compactly generated Banach spaces, Compositio Math. 28 (1974), 83-111.

[13] P. Roy, Nonequality of dimensions for metric spaces, Trans. Amer. Math. Soc. 134 (1968), 117-132.

[14] N. V. Veličko, The cardinality of open coverings of topological spaces, Fund. Math. 80 (1973), 271-282 (in Russian).

Department of Mathematics

University of the Aegean

83 200, Karlovassi, Samos, Greece

E-mail: mcha@aegean.gr

Received 16 September 2003;

in revised form 14 June 2004 